\title{
Special issue on selected papers from the IEEE Latin America on Communications (LATINCOM)
}

\author{
Fabrizio Granelli ${ }^{1}$. Oscar Caicedo ${ }^{2} \cdot$ Gustavo B. Figueiredo $^{3} \cdot$ Nelson L. S. da Fonseca $^{4}$ \\ Accepted: 15 December 2021 / Published online: 7 February 2022 \\ (c) The Author(s), under exclusive licence to Springer Science+Business Media, LLC, part of Springer Nature 2022
}

The IEEE Latin America on Communications (LATINCOM), an IEEE Communications Society conference, is the premier event on communications and networks in Latin America. This special issue contains selected papers from the 2019 and 2020 LATINCOM editions. The papers in this issue are extended version of the conference papers and were reviewed by independent reviewers.

In "Novel Cluster Partitioning Models for Visible Light Communication Networks", Adasme et al. consider the problem of clustering nodes in a wireless visible light communication (VLC) network while simultaneously forming. The idea is to form clusters of nodes while simultaneously providing connectivity between them and maximize the total power received and total residual energy nodes of the network. A mixed-integer linear and quadratic programming model based on classical combinatorial optimization problems is proposed to solve the stated problem.

In "Impact of Diversity Combining Schemes in a MultiCell VLC System with Angle Diversity Receivers", Román Cãnizares et al. analyze and compare the use and benefits of several combining schemes to create diversity at the optical receiver in a multi-cell indoor visible light communication (VLC) system. The schemes at the receiver side are studied and implemented: Select Best Combining, Equal Gain Combining, Generalized Selection Combining, and Maximum Ratio Combining are evaluated. These techniques, in conjunction with a pyramidal angle diversity receiver (ADR), enhance the performance of the VLC system by selecting or combining the received optical signals.

\footnotetext{
Nelson L. S. da Fonseca

nfonseca@ic.unicamp.br

University of Trento, Trento, Italy

University of Cauca, Popayán, Colombia

Federal University of Bahia, Salvador, Brazil

State University of Campinas, Campinas, Brazil
}

In "Performance Analysis of IEEE 802.15.7-Based Visible Light Communication Systems in Underground Mine Environments", Palacios Játiva propose a viable solution to complement the systems with a communication infrastructure based on visible light communication (VLC) to cope with irregular features of underground mining (UM) tunnels and environment electromagnetic interference. The authors study and characterize the physical factors and external phenomena of UM tunnels, as well as the orientation of optical components that affect the performance of VLC systems.

In "Performance evaluation of multi-ONU Customers in Ethernet Passive Optical Networks", Ciceri et al. assess the performance of the Interleaved Polling with Adaptive Cycle Time with Multi-ONU SLAs support (MOS-IPACT) algorithm, a dynamic bandwidth allocation (DBA) algorithm recently proposed to enable redistribution of unused guaranteed bandwidth among ONUs belonging to the same multi-ONU customer. In particular, the article proposes an analytical model for MOS-IPACT and evaluates the benefits of exploiting bandwidth sharing among ONUs of multiONU customers.

In "DBA Algorithm for the Support of Multi-tiered Bandwidth Guarantee in Ethernet Passive Optical Networks", Ciceri et al. propose a novel DBA scheme for supporting prioritized scheduling. The proposal assures bandwidth to the different customers and prioritizes the excess bandwidth distribution among subgroups of ONUs of the same multiONU customer. The proposal allows providers to share bandwidth among ONUs of the same multi-ONU customers while supporting services with different priorities.

In "Deep Recurrent Neural Network for Optical Fronthaul Dimensioning and Proactive vBBU Placement in CF-RAN", Santos et al. propose a new heuristic for the placement of BaseBand Units and VPON assignment in Hybrid Radio Access Networks. The approach relies on traffic predictions calculated using a Deep Recurrent Neural Network to absorb the tidal effect of mobile network traffic. With that, the algorithm receives an accurate input about the future traffic and 
can make decisions in advance. Simulation results of this method show strong similarity with those obtained by optimum solutions generated by ILP without the long execution times that characterize the latter.

In "Defining Amplifier's Gain to Maximize the Transmission Rate in Optical Systems Using Evolutionary Algorithms and Swarm Intelligence", Lima et al. propose to improve network efficiency is to use dynamic approaches like Adaptive Control of Operating Point, which consists of autonomously choosing the best operating point for optical amplifiers on the link, thus providing the best configuration concerning quality of transmission. Unlike the previous works that focused on optimizing optical signal-to-noise ratio, the proposal focuses on maximizing the transmission rate. Five different evolutionary and swarm-based algorithms in the search for maximizing the transmission rate in optical links are compared. Differential evolution provided the best results in the analyzed scenarios.

In "A Number-Theoretic Framework for the Mitigation of Fragmentation Loss in Elastic Optical Links", Waldman et al. address the problem of minimizing total slot idle time in assigning spectrum to a 2-class traffic, considering both incremental and dynamic arrival and permanence rules. Deadlock avoidance under incremental traffic is shown to be possible with the use of non-greedy spectrum assignment policies in some link states which are identified from knowledge of the connection request sizes, keeping total idleness finite and minimal. Moreover, the concept of deadlock avoidance is extended to dynamic traffic with the purpose of proposing an algorithm that mitigates fragmentation losses with appropriate greedy traffic-aware assignment policies.

The Guest Editors would like to thank the contributing authors for their efforts and hope that the reader will find in this issue suggestions and fresh ideas for research in photonic communications and networks.

Fabrizio Granelli (Lead Guest Editor), University of Trento, Italy.

Oscar M. Caicedo Rendon, Universida del Cauca, Colombia.

Gustavo B. Figueiredo, Federal University of Bahia, Brazil.

Nelson L. S. da Fonseca, State University of Campinas, Brazil.

Publisher's Note Springer Nature remains neutral with regard to jurisdictional claims in published maps and institutional affiliations. 